TRANSACTIONS OF THE

AMERICAN MATHEMATICAL SOCIETY

Volume 353, Number 4, Pages 1455-1468

S 0002-9947(00)02629-5

Article electronically published on October 11, 2000

\title{
$K 3$ SURFACES OF GENUS 8 AND VARIETIES OF SUMS OF POWERS OF CUBIC FOURFOLDS
}

\author{
ATANAS ILIEV AND KRISTIAN RANESTAD
}

\begin{abstract}
The main result of this paper is that the variety of presentations of a general cubic form $f$ in 6 variables as a sum of 10 cubes is isomorphic to the Fano variety of lines of a cubic 4-fold $F^{\prime}$, in general different from $F=Z(f)$.

A general $K 3$ surface $S$ of genus 8 determines uniquely a pair of cubic 4folds: the apolar cubic $F(S)$ and the dual Pfaffian cubic $F^{\prime}(S)$ (or for simplicity $F$ and $F^{\prime}$ ). As Beauville and Donagi have shown, the Fano variety $\mathcal{F}_{F^{\prime}}$ of lines on the cubic $F^{\prime}$ is isomorphic to the Hilbert scheme $\mathrm{Hilb}_{2} S$ of length two subschemes of $S$. The first main result of this paper is that $\mathrm{Hilb}_{2} S$ parametrizes the variety $\operatorname{VSP}(F, 10)$ of presentations of the cubic form $f$, with $F=Z(f)$, as a sum of 10 cubes, which yields an isomorphism between $\mathcal{F}_{F^{\prime}}$ and $\operatorname{VSP}(F, 10)$. Furthermore, we show that $\operatorname{VSP}(F, 10)$ sets up a $(6,10)$ correspondence between $F^{\prime}$ and $\mathcal{F}_{F^{\prime}}$. The main result follows by a deformation argument.
\end{abstract}

\section{Pfaffian And apolar CUbiC 4-Folds ASSOCIATED TO $K 3$ SURFACES OF GENUS 8}

1.1. Let $V$ be a 6-dimensional vector space over C. Fix a basis $e_{0}, \ldots, e_{5}$ for $V$; then $e_{i} \wedge e_{j}$ for $0 \leq i<j \leq 5$ form a basis for the Plücker space $\wedge^{2} V$ of 2-dimensional subspaces in $V$ or lines in $\mathbf{P}^{5}=\mathbf{P}(V)$. We associate to a 2-vector $g=\sum_{i<j} a_{i j} e_{i} \wedge e_{j} \in \wedge^{2} V$ a skewsymmetric matrix $M(g)=\left(a_{i j}\right)$, with $a_{j i}=-a_{i j}$. With Plücker coordinates $x_{i j}$, the embedding of the Grassmannian $G=\mathbf{G}(2, V)$ in $\mathbf{P}^{14}=\mathbf{P}\left(\Lambda^{2} V\right)$ is then precisely the locus of rank 2 skewsymmetric $6 \times 6$ matrices

$$
M=\left(\begin{array}{cccccc}
0 & x_{01} & x_{02} & x_{03} & x_{04} & x_{05} \\
-x_{01} & 0 & x_{12} & x_{13} & x_{14} & x_{15} \\
-x_{02} & -x_{12} & 0 & x_{23} & x_{24} & x_{25} \\
-x_{03} & -x_{13} & -x_{23} & 0 & x_{34} & x_{35} \\
-x_{04} & -x_{14} & -x_{24} & -x_{34} & 0 & x_{45} \\
-x_{05} & -x_{15} & -x_{25} & -x_{35} & -x_{45} & 0
\end{array}\right) .
$$

Since the sum of two rank 2 matrices has rank at most 4, and any rank 4 skewsymmetric matrix is the sum of two rank 2 skewsymmetric matrices, the secant variety of $G$ is the cubic hypersurface $K$ defined by the $6 \times 6$ Pfaffian $m$ of the matrix $M$. The dual variety of $G$ in $\check{\mathbf{P}}^{14}=\mathbf{P}\left(\wedge^{2} V^{*}\right)$ is a cubic hypersurface $K^{*} \cong K$ (cf. [10]). $K^{*}$ is the secant variety of $G^{*}=\mathbf{G}(V, 2)$, the Grassmannian of rank 2 quotient spaces of $V$, and of course $G^{*} \cong G$.

Received by the editors July 5, 1999.

2000 Mathematics Subject Classification. Primary 14J70; Secondary 14M15, 14N99. 
1.2. A general $K 3$ surface $S$ with Picard group generated by a linebundle $H$ of degree $H^{2}=14$ is embedded via $|H|$ into the Grassmannian $G^{*}=\mathbf{G}(V, 2)$. In fact $S$ is the intersection of $G^{*}$ with a linear space $L_{S}$ of dimension 8 in $\check{\mathbf{P}}^{14}=\mathbf{P}\left(\wedge^{2} V^{*}\right)$ (cf. [7]). The dual space $P_{S}=L_{S}^{\frac{1}{S}} \subset \mathbf{P}^{14}$ is 5-dimensional, so $P_{S}$ intersects the dual variety $K$ of $G^{*}$ in a Pfaffian cubic 4 -fold which we denote by $F^{\prime}(S)$ (or simply $\left.F^{\prime}\right)$.

1.3. The Plücker embedding of the Grassmannian $G=\mathbf{G}(2, V)$ in $\mathbf{P}^{14}$ is arithmetically Gorenstein. The homogeneous coordinate ring $A_{G}$ has syzygies, easily computed with MACAULAY ([6]),

$\begin{array}{ccccccc}1 & - & - & - & - & - & - \\ - & 15 & 35 & 21 & - & - & - \\ - & - & - & 21 & 35 & 15 & - \\ - & - & - & - & - & - & 1\end{array}$

in MACAULAY notation. The Grassmannian variety has dimension 8 , so $P_{S}=$ $L_{S}^{\perp}$, defined by the linear forms $h_{0}, \ldots, h_{8}$ in $\mathbf{P}^{14}$, does not intersect $G$, and the quotient $A=A_{G} /\left(h_{0}, \ldots, h_{8}\right)$ is an Artinian Gorenstein ring. Its Hilbert function is $(1,6,6,1)$ with socledegree 3 , so $A$ is the apolar Artinian Gorenstein ring $A^{F}$ for some cubic hypersurface $F=F(S) \subset \check{P}_{S}$. More precisely, the dual socle generator of $A$ is a cubic form $f$, defined up to a scalar by $A$, with $F=Z(f)$. We call $F=F(S)$ the apolar cubic 4-fold of $S$.

1.4. Lemma. There is a 19-dimensional family of cubic 4-folds $F$ whose apolar Artinian Gorenstein ring is a quotient of $A_{G}$.

Proof. Macaulay (cf. [5]) showed that there is a $1: 1$ correspondence between hypersurfaces of degree $d$ and graded Artinian Gorenstein rings generated in degree 1 with socledegree $d$ (cf. 3.3 below). Now, an isomorphism between such rings is of course induced by a linear transformation on the generators. In our setting any such linear transformation is again induced by an automorphism of $G^{*}$ and correspondingly of $G$. The isomorphism classes of general $K 3$ surfaces of genus 8 correspond precisely to orbits of 8-dimensional subspaces $L$ (cf. [7). There is a 19-dimensional family of $K 3$ surfaces of genus 8 , so the lemma follows.

1.5. Remark. The apolar cubic $F=F(S)$ is in general not a Pfaffian cubic.

In fact the Pfaffian cubics also form a 19-dimensional family of cubic 4-folds, and the above correspondence determines a birationality between the family of Pfaffian cubics and the family of apolar cubics $F$. On the other hand, computing the apolar quadrics to a Pfaffian cubic with [6] (cf. [3.2 for apolarity), it can readily be checked that there are in general no quadratic relations between them, while the apolar quadrics to a cubic $F$ have nine quadratic relations: As we shall see in the next section, the apolar quadrics define the restriction to the 5-space $P=P_{S}=L_{S}^{\perp}$ of the Cremona transformation defined by all quadrics through $G$. The inverse Cremona transformation is defined by quadrics again, and since $P$ has codimension 9 in $\mathbf{P}^{14}$, there are at least nine quadrics containing the image of $P$, i.e. at least nine quadratic relations between the apolar quadrics.

1.6. Problem. Find an alternative description of the apolar cubic 4 -folds $F$.

The main results of this paper are stated and proved in the third section. Before that we state some properties of the Cremona transformation defined by the 
Pfaffians of the matrix $M$, which we collect in the next section. These properties are also of independent interest.

1.7. Notation. The kernel of an element $\alpha \in \wedge^{2} V^{*}$ is the subspace

$$
\text { ker } \alpha=\{v \in V \mid \alpha(u \wedge v)=0, \forall u \in V\} \text {. }
$$

A subspace $U \subset V$ is Lagrangian with respect to $\alpha$ if $\left.\alpha\right|_{U} \equiv 0$. Similarly we define for $g \in \wedge^{2} V$ the kernel ker $g \subset V^{*}$ and Lagrangian subspaces with respect to $g$ in $V^{*}$. For any $g \in \wedge^{2} V$, we denote by $|g|$ the subspace $(\operatorname{ker} g)^{\perp} \subset V$, and call it the support of $g$. For $g \in G$ the support $|g|$ is of course the rank 2 subspace of $V$ represented by $g$. The rank of the support $|g|$ clearly equals the rank of $g$. Similarly we define the support of $\alpha$ in $V^{*}$.

\section{Geometry of $\mathbf{G}(2, V)$ and its associated Cremona transformation}

2.1. In the Plücker coordinates $x_{i j}$, the equations of $G=\mathbf{G}(2, V)$ are the $4 \times 4$ Pfaffians of the matrix $M$. Let $q_{i j}=\operatorname{Pfaff}_{i j} M$ be the $4 \times 4$ Pfaffians for $0 \leq i<$ $j \leq 5$ and let $m=$ Pfaff $M$ be the $6 \times 6$ Pfaffian of $M$. While

$$
\begin{aligned}
m & =x_{05} x_{14} x_{23}-x_{04} x_{15} x_{23}-x_{05} x_{13} x_{24} \\
& +x_{03} x_{15} x_{24}+x_{04} x_{13} x_{25}-x_{03} x_{14} x_{25} \\
& +x_{05} x_{12} x_{34}-x_{02} x_{15} x_{34}+x_{01} x_{25} x_{34} \\
& -x_{04} x_{12} x_{35}+x_{02} x_{14} x_{35}-x_{01} x_{24} x_{35} \\
& +x_{03} x_{12} x_{45}-x_{02} x_{13} x_{45}+x_{01} x_{23} x_{45}
\end{aligned}
$$

the quadrics $q_{i j}$ are

$$
\begin{array}{ll}
q_{45}=x_{03} x_{12}-x_{02} x_{13}+x_{01} x_{23} & q_{35}=x_{04} x_{12}-x_{02} x_{14}+x_{01} x_{24} \\
q_{34}=x_{05} x_{12}-x_{02} x_{15}+x_{01} x_{25} & q_{25}=x_{04} x_{13}-x_{03} x_{14}+x_{01} x_{34} \\
q_{24}=x_{05} x_{13}-x_{03} x_{15}+x_{01} x_{35} & q_{23}=x_{05} x_{14}-x_{04} x_{15}+x_{01} x_{45} \\
q_{15}=x_{04} x_{23}-x_{03} x_{24}+x_{02} x_{34} & q_{14}=x_{05} x_{23}-x_{03} x_{25}+x_{02} x_{35} \\
q_{13}=x_{05} x_{24}-x_{04} x_{25}+x_{02} x_{45} & q_{12}=x_{05} x_{34}-x_{04} x_{35}+x_{03} x_{45} \\
q_{05}=x_{14} x_{23}-x_{13} x_{24}+x_{12} x_{34} & q_{04}=x_{15} x_{23}-x_{13} x_{25}+x_{12} x_{35} \\
q_{03}=x_{15} x_{24}-x_{14} x_{25}+x_{12} x_{45} & q_{02}=x_{15} x_{34}-x_{14} x_{35}+x_{13} x_{45} \\
q_{01}=x_{25} x_{34}-x_{24} x_{35}+x_{23} x_{45} &
\end{array}
$$

Notice that $(-1)^{i+j-1} q_{i j}$ is precisely the partial of $m$ with respect to $x_{i j}$, i.e.,

$$
3 m=\sum_{0 \leq i<j \leq 5}(-1)^{i+j-1} x_{i j} q_{i j}
$$

The Pfaffians $q_{i j}$ define a Cremona transformation (cf. [3])

$$
\varphi: \mathbf{P}^{14}--->\mathbf{P}^{14}
$$

In fact

$$
q_{i j}\left(q_{s t}\right)=m x_{i j}
$$

so the Cremona transformation is its own inverse. The Cremona transformation contracts precisely all secants to $G$. The exceptional divisor lying over $G$ in the Cremona transformation is mapped to a cubic hypersurface $K^{\prime}$, the secant variety of a variety $G^{\prime}$, which in turn is isomorphic to $G$. 
2.2. In terms of 2-vectors $g \in \wedge^{2} V$ this Cremona transformation is the composition

$$
\varphi: \wedge^{2} V \rightarrow \wedge^{4} V \rightarrow \wedge^{2} V^{*}, \quad g \mapsto g \wedge g \mapsto g \wedge g-,
$$

where the last map is the natural isomorphism (canonical up to scalars). Therefore the target space of the Cremona transformation is naturally identified with $\check{\mathbf{P}}^{14}$. It is a morphism on the complement of $G=\mathbf{G}(2, V)$, it is birational on the complement of $K$, while $K \backslash G$ is mapped to $G^{*}=\mathbf{G}(V, 2)$. In fact, $g \wedge g=0$ for $g \in G$, while $\operatorname{ker} \varphi(g)=|g|$ when $g \in K \backslash G$.

The fiber of $\varphi$ over a point $\alpha \in G^{*}$ is

$$
\varphi^{-1}(\alpha)=\left\{g \in \mathbf{P}\left(\wedge^{2} V\right)|| g \mid=\operatorname{ker} \alpha\right\}=\mathbf{P}\left(\wedge^{2} \operatorname{ker} \alpha\right),
$$

a 5 -dimensional space which intersects $G$ in a quadric hypersurface $\mathbf{G}(2, \operatorname{ker} \alpha)$.

2.3. The preimage under $\varphi$ of a line in $G^{*}$ is a rational scroll ruled in 5-dimensional spaces. For this, first note that the points where only the quadrics $q_{01}$ and $q_{02}$ are nonzero are mapped to a line on $G^{*}$. On the other hand, by inspection, all the quadrics $q_{i j}$, except $q_{01}$ and $q_{02}$, vanish on the union of $G$ and the cubic scroll defined by the $2 \times 2$ minors of

$$
\left(\begin{array}{lll}
x_{13} & x_{14} & x_{15} \\
x_{23} & x_{24} & x_{25}
\end{array}\right)
$$

inside the 8-dimensional space $Z\left(x_{01}, x_{02}, x_{03}, x_{04}, x_{05}, x_{12}\right)$. By homogeneity on $G^{*}$ the preimage of any line is a 6 -fold cubic scroll.

2.4. There are two kinds of planes in $G^{*}$. First we have the planes representing all lines in a plane, and second we have the planes representing all lines through a point in a 3 -space. The points where all the quadrics $q_{i j}$ except $q_{01}, q_{02}$ and $q_{12}$ vanish are all mapped to a plane in $G^{*}$ of the first kind. On the other hand, by inspection, all these quadrics vanish on the union of $G$ and a 7-dimensional subvariety of degree 6 defined by the $2 \times 2$ minors of

$$
\left(\begin{array}{lll}
x_{03} & x_{04} & x_{05} \\
x_{13} & x_{14} & x_{15} \\
x_{23} & x_{24} & x_{25}
\end{array}\right)
$$

inside the 11-dimensional space $Z\left(x_{01}, x_{02}, x_{12}\right)$. By homogeneity the preimage under $\varphi$ of any plane of the first kind is a 7 -fold of degree 6 in a $\mathbf{P}^{11}$. The points where all the quadrics $q_{i j}$ except $q_{01}, q_{02}$ and $q_{03}$ vanish are all mapped to a plane in $G^{*}$ of the second kind. On the other hand, by inspection, all these quadrics vanish on the union of $G^{*}$ and the 7-dimensional complete intersection of the two quadrics $q_{04}$ and $q_{05}$ inside the 9 -dimensional space $Z\left(x_{01}, x_{02}, x_{03}, x_{04}, x_{05}\right)$. By homogeneity the preimage under $\varphi$ of any plane of the second kind is a 7 -fold complete intersection of two quadrics in a $\mathbf{P}^{9}$.

2.5. Next we consider a tangent space to $G$. Without loss of generality we may consider the line spanned by $L_{01}=\left\langle e_{0}, e_{1}\right\rangle \subset \mathbf{P}(V)$ corresponding to the point $p_{01}=(1,0, \ldots, 0)$ on $G$. Let

$$
N_{01}=\left(\begin{array}{cccc}
x_{02} & x_{03} & x_{04} & x_{05} \\
x_{12} & x_{13} & x_{14} & x_{15}
\end{array}\right)
$$


2.6. Lemma. $N_{01}$ has rank 1 on $G$ precisely at the points which correspond to lines which meet $L_{01}$. In fact the tangent space to $G$ at $p_{01}$ is defined by $x_{i j}=$ $0,2 \leq i<j \leq 5$, and the $2 \times 2$ minors of $N_{01}$ define the contact cone inside this tangent space.

Proof. When $x_{i j}=0$ for $2 \leq i<j \leq 5$, then the Plücker quadrics reduce to the minors of $N_{01}$. On the other hand, when this matrix has rank 1, i.e.,

$$
\alpha\left(x_{02}, x_{03}, x_{04}, x_{05}\right)+\beta\left(x_{12}, x_{13}, x_{14}, x_{15}\right)=(0,0,0,0),
$$

then it is the Grassmannian point of the line

$$
\left(\beta e_{0}+\alpha e_{1}\right) \wedge\left(x_{02} e_{2}+x_{03} e_{3}+x_{04} e_{4}+x_{05} e_{5}\right),
$$

which is a general line which meets $L_{01}$.

Next, we associate to each line in $K$ a rank 4 subspace of $V^{*}$ (cf. [2]).

2.7. Lemma. Let $l \in K$ be a line that does not intersect $G$. Then there is a unique 4-space $V^{*}(l) \subset V^{*}$, which is Lagrangian with respect to every element of $l$.

Proof. Let $g, g^{\prime}$ be two points that span $l$. Then $\operatorname{ker} g$ and $\operatorname{ker} g^{\prime}$ are both 2dimensional subspaces of $V^{*}\left(\operatorname{rank} g=\operatorname{rank} g^{\prime}=4\right)$. Since $l$ does not intersect $G$, the two kernels span a 4-space. We may choose a basis $\left\langle e_{0}^{*}, \ldots, e_{5}^{*}\right\rangle$ for $V^{*}$ such that $\left\langle e_{0}^{*}, e_{1}^{*}\right\rangle$ and $\left\langle e_{4}^{*}, e_{5}^{*}\right\rangle$ are the two kernels. Thus the skewsymmetric matrices $M(g)$ and $M\left(g^{\prime}\right)$ have coefficients

$$
M(g)=\left(a_{i j}\right), a_{i i}=0, a_{i j}=-a_{j i}, a_{0 j}=a_{1 j}=0
$$

and

$$
M\left(g^{\prime}\right)=\left(b_{i j}\right), b_{i i}=0, b_{i j}=-b_{j i}, b_{4 j}=b_{5 j}=0 .
$$

Let $h=s g+t g^{\prime}$ be a general point on $l$; then $M(h)=\left(s a_{i j}+t b_{i j}\right)$. Now $l \subset K$, so $M(h)$ has rank 4 (independant of $s$ and $t$ ). Therefore Pfaff $M(h)=$ $3 s t\left(t a_{45} \mathrm{Pfaff}_{45} M\left(g^{\prime}\right)+s b_{01} \mathrm{Pfaff}_{01} M(g)\right)=0$ for every $s$ and $t$. But since $M(g)$ and $M\left(g^{\prime}\right)$ have rank 4, both Pfaff $01 M(g)$ and Pfaff $45 M\left(g^{\prime}\right)$ are nonzero, so $a_{45}=$ $b_{01}=0$. This means that that the 4-space $\left\langle e_{0}, e_{1}, e_{4}, e_{5}\right\rangle$ is Lagrangian for all $h \in l$. On the other hand, any common Lagrangian 4-space has to contain ker $g$ and $\operatorname{ker} g^{\prime}$, so it is unique.

Finally, we investigate certain subvarieties associated to secant lines to $G^{*}$. To a proper secant line $l^{*}$, we associate a quadric surface $Q\left(l^{*}\right) \subset G^{*}$. If the secant line $l^{*}$ intersects $G^{*}$ in two points, the quadric surface $Q\left(l^{*}\right)$ parametrizes all lines that intersect the lines represented by these two points. Notice that the support $|\alpha|$ is common for every rank 4 element $\alpha \in l^{*}$; we denote this by $U\left(l^{*}\right)$. Thus $Q\left(l^{*}\right) \subset$ $\mathbf{G}\left(2, U\left(l^{*}\right)\right) \subset \mathbf{P}\left(\wedge^{2} U\left(l^{*}\right)\right)$. Since $U\left(l^{*}\right)$ is 4-dimensional, $\mathbf{G}\left(2, U\left(l^{*}\right)\right)$ is a smooth quadric hypersurface. Polarity with respect to this quadric defines a correlation $p_{U}: \mathbf{P}\left(\wedge^{2} U\left(l^{*}\right)\right) \rightarrow \mathbf{P}\left(\wedge^{2} U\left(l^{*}\right)^{*}\right)$. Now $l^{*} \subset \mathbf{P}\left(\wedge^{2} U\left(l^{*}\right)\right)$, so $p_{U}\left(l^{*}\right)^{\perp} \subset \mathbf{P}\left(\wedge^{2} U\left(l^{*}\right)\right)$ is a 3 -space $P\left(l^{*}\right)$, the span of $Q\left(l^{*}\right)$.

When $l^{*} \subset \mathbf{P}\left(\wedge^{2} U\right)$ for some 4-dimensional subspace $U \subset V^{*}$, we denote by $P_{U}\left(l^{*}\right)$ the 3 -space $p_{U}\left(l^{*}\right)^{\perp} \subset \mathbf{P}\left(\wedge^{2} U\right)$ polar to $l^{*}$ with respect to the quadric $\mathbf{G}(2, U)$. The pairs $\left(l^{*}, Q\left(l^{*}\right)\right)$ therefore fit into the following incidence:

$$
I_{Q}=\left\{\left(l^{*}, Q\right) \mid \exists U \subset V^{*}, \operatorname{dim} U=4, l^{*} \subset \mathbf{P}\left(\wedge^{2} U\right), Q=P_{U}\left(l^{*}\right) \cap G^{*}\right\}
$$


2.8. Lemma. Let $\left(l^{*}, Q\right) \in I_{Q}$. Then $l^{*}$ is a secant line to $G^{*}$, a tangent line to $G^{*}$, or is contained in $G^{*}$. It is a secant line if and only if $Q$ is smooth. It is a tangent line if and only if $Q$ has rank 3 . It is contained in $G^{*}$ if and only if $Q$ has rank 2. The projection onto the second factor is onto the variety of quadric surfaces in $G^{*}$, whose span is not contained in $G^{*}$. The first projection is one to one except over the lines in $G^{*}$, where each fiber is a $\mathbf{P}^{2}$.

Proof. The definition above of $Q\left(l^{*}\right)$ clearly extends to tangent lines: If $l^{*}$ is a tangent line to $G^{*}$, then $|\alpha|=U$ is constant for every rank 4 element $\alpha \in l^{*}$, and $Q\left(l^{*}\right)$ is a singular $\mathbf{P}^{3}$ section of $\mathbf{G}(2, U)$. If $l^{*}$ is a line in $G^{*}$, then $\bigcup_{\alpha}|\alpha|$ for $\alpha \in l^{*}$ is a 3-dimensional subspace $U_{0} \subset V^{*}$. Any 4-space $U \supset U_{0}$ gives rise to a quadric $Q \subset G^{*}$ of rank 2. One of the planes of $Q$ is common for all $U \supset U_{0}$; it is the plane $\mathbf{G}\left(2, U_{0}\right)$. In all cases it is straightforward to check that the span of the quadric surface is polar to $l^{*}$ with respect to the quadric $\mathbf{G}(2, U)$.

Thus we are left to prove that the second projection is onto the family of quadric surfaces, whose span is not contained in $G^{*}$.

Let $Q$ be a quadric surface of this kind. First note that the span $P_{Q}$ of $Q$ is contained in $K^{*}$. The restriction of the inverse Cremona transformation to $P_{Q}$ is clearly the constant map onto some point on $G$. Thus $P_{Q}$ is contained in fiber of this inverse transformation. By 2.2 the whole fiber is a $\mathbf{P}\left(\wedge^{2} U\right)$ which intersects $G^{*}$ in the rank 6 quadric $\mathbf{G}(2, U)$. Therefore the intersection of this quadric with a 3-space has rank at least 2. Furthermore, $P_{Q}=P_{U}\left(l^{*}\right) \subset \mathbf{P}\left(\wedge^{2} U\right)$ for some $l^{*} \subset \mathbf{P}\left(\wedge^{2} U\right)$. The lemma now follows.

2.9. Lemma. Let $Q$ be a quadric surface in $G^{*}$, whose span is not contained in $G^{*}$, and let $l^{*}$ be a line in $G^{*}$ such that $\left(l^{*}, Q\right)$ belongs to the incidence $I_{Q}$. Then the preimage $\varphi^{-1}(Q)$ under the Cremona transformation is the intersection of two rational quartic scrolls in a rank 4 quadric hypersurface inside $\left(l^{*}\right)^{\perp}$. In particular, it has degree 10 and codimension 5 inside $\left(l^{*}\right)^{\perp}$.

Proof. For this, consider first the secant line $l^{*}$ of $G^{*}$ spanned by the points with coordinates $y_{s t}=0$ for $(s t) \neq(01)$ and $(s t) \neq(23)$ respectively. Then $Q\left(l^{*}\right)=Z\left(y_{02} y_{13}-y_{12} y_{03}\right)$ inside the 3 -space $P\left(l^{*}\right)=Z\left(y_{s t} \mid(s t) \notin\{02,03,12,13\}\right)$. Therefore the preimage of $Q\left(l^{*}\right)$ under the Cremona transformation is defined by the 11 quadrics

$$
V(01,23)=\left\langle q_{s t} \mid(s t) \notin\{02,03,12,13\}\right\rangle .
$$

These quadrics vanish on the union of $G$ and

$$
Z(01,23)=Z(V(01,23)) \cap Z\left(x_{01}, x_{23}\right) .
$$

By inspection, $Z(01,23)$ is the locus inside the hyperplanes $Z\left(x_{01}\right)$ and $Z\left(x_{23}\right)$ where the matrices

$$
N_{01}=\left(\begin{array}{cccc}
x_{02} & x_{03} & x_{04} & x_{05} \\
x_{12} & x_{13} & x_{14} & x_{15}
\end{array}\right) \text { and } N_{23}=\left(\begin{array}{llll}
x_{02} & x_{12} & x_{24} & x_{25} \\
x_{03} & x_{13} & x_{34} & x_{35}
\end{array}\right)
$$

both drop rank. The invariants of $Z(01,23)$ are easily computed in MACAULAY [6]. The matrices $N_{01}$ and $N_{23}$ each drop rank on a rational quartic scroll of codimension 3 . The two matrices have the $2 \times 2$ submatrix $\left(\begin{array}{ll}x_{02} & x_{03} \\ x_{12} & x_{13}\end{array}\right)$ in common, so $Z(01,23)$ is the intersection of two rational normal quartic scrolls inside a rank 
4 quadric. For the degree we note that this subvariety is a degeneration of the intersection of two codimension 2 cycles of bidegree $(1,3)$ on a rank 6 quadric. Thus the degree is 10 .

By homogeneity 2.9 follows for smooth quadric surfaces. The computations for quadric surfaces of rank 3 and 2 are quite similar and straightforward to check.

\section{The VARIETy of Sums of POWers $\operatorname{VSP}(F, 10)$}

3.1. For a homogeneous polynomial $f$ of degree $d$ in $n+1$ variables, which define the hypersurface $F=Z(f) \subset \mathbf{P}^{n}$, we define the variety of sums of powers as the closure

$$
=\frac{V P(F, s)}{\left\{\left\{\left\langle l_{1}\right\rangle, \ldots,\left\langle l_{s}\right\rangle\right\} \in H_{i l b_{s}}\left(\check{\mathbf{P}}^{n}\right) \mid \exists \lambda_{i} \in \mathbf{C}: f=\lambda_{1} l_{1}^{d}+\ldots+\lambda_{s} l_{s}^{d}\right\}}
$$

of the set of powersums presenting $F$ in the Hilbert scheme (cf. [8]). We study these powersums using apolarity.

3.2. Apolarity (cf. [8]). Consider $R=\mathbf{C}\left[x_{0}, \ldots, x_{n}\right]$ and $T=\mathbf{C}\left[\partial_{0}, \ldots, \partial_{n}\right] . T$ acts on $R$ by differentiation:

$$
\partial^{\alpha}\left(x^{\beta}\right)=\alpha !\left(\begin{array}{l}
\beta \\
\alpha
\end{array}\right) x^{\beta-\alpha}
$$

if $\beta \geq \alpha$, and 0 otherwise. Here $\alpha$ and $\beta$ are multi-indices, $\left(\begin{array}{c}\beta \\ \alpha\end{array}\right)=\prod\left(\begin{array}{c}\beta_{i} \\ \alpha_{i}\end{array}\right)$, and so on. In particular we have a perfect pairing between forms of degree $d$ and homogeneous differential operators of order $d$. Note that the polar of a form $f \in R$ in a point $a \in \mathbf{P}^{n}$ is given by $P_{a}(f)$ for $a=\left(a_{0}, \ldots, a_{n}\right)$ and $P_{a}=\sum a_{i} \partial_{i} \in T$.

One can interchange the roles of $R$ and $T$ by defining

$$
x^{\beta}\left(\partial^{\alpha}\right)=\beta !\left(\begin{array}{c}
\beta \\
\alpha
\end{array}\right) \partial^{\alpha-\beta} .
$$

With this notation we have, for forms of degree $n$,

$$
P_{a}^{d}(f)=f\left(P_{a}^{d}\right)=d ! f(a) .
$$

Moreover,

$$
f\left(P_{a}^{m}\right)=0 \Longleftrightarrow f(a)=0
$$

if $m \geq d$. More generally, we say that homogeneous forms $f \in R$ and $D \in T$ are apolar if $f(D)=D(f)=0$ (According to Salmon (1885) [9], the term was coined by Reye.)

Apolarity allows us to define Artinian Gorenstein graded quotient rings of $T$ via forms: For $f$ a homogeneous form of degree $d$ and $F=Z(f) \subset \mathbf{P}^{n}$, define

$$
F^{\perp}=f^{\perp}=\{D \in T \mid D(f)=0\}
$$

and

$$
A^{F}=T / F^{\perp}
$$

An obvious but useful identity in apolarity is

$$
\left(f^{\perp}: D\right)=D(f)^{\perp}
$$

for any homogeneous $D \in T$.

The socle of $A^{F}$ is in degree $d$. Indeed, $P_{a}(D(f))=0 \forall P_{a} \in T_{1} \Longleftrightarrow D(f)=$ 0 or $D \in T_{d}$. In particular, the socle of $A^{F}$ is 1 -dimensional, and $A^{F}$ is indeed 
Gorenstein and is called the apolar Artinian Gorenstein ring of $F \subset \mathbf{P}^{n}$. Conversely, for a graded Gorenstein ring $A=T / I$ with socledegree $d$, multiplication in $A$ induces a linear form $f: \operatorname{Sym}_{d}\left(T_{1}\right) \rightarrow \mathbf{C}$ which can be identified with a homogeneous polynomial $f \in R$ of degree $d$. This proves:

3.3. Lemma (Macaulay, [5]). The map $F \mapsto A^{F}$ is a bijection between hypersurfaces $F=V(f) \subset \mathbf{P}^{n}$ of degree $d$ and graded Artinian Gorenstein quotient rings $A=T / I$ of $T$ with socledegree $d$.

In the following we identify $R$ with the homogeneous coordinate ring of $\mathbf{P}^{n}$ and $T$ with the homogeneous coordinate ring of the dual space $\check{\mathbf{P}}^{n}$. Let $F=Z(f) \subset \mathbf{P}^{n}$ denote a hypersurface of degree $d$. We call a subscheme $\Gamma \subset \check{\mathbf{P}}^{n}$ apolar to $F$, if the homogeneous ideal $I_{\Gamma} \subset F^{\perp} \subset T$.

3.4. Lemma. Let $l_{1}, \ldots, l_{s}$ be linear forms in $R$, and let $L_{i} \in \check{\mathbf{P}}^{n}$ be the corresponding points in the dual space. Then $f=\lambda_{1} l_{1}^{d}+\ldots+\lambda_{s} l_{s}^{d}$ for some $\lambda_{i} \in \mathbf{C}^{*}$ if and only if $\Gamma=\left\{L_{1}, \ldots, L_{s}\right\} \subset \check{\mathbf{P}}^{n}$ is apolar to $F=Z(f)$.

Proof. Assume $f=\lambda_{1} l_{1}^{d}+\ldots+\lambda_{s} l_{s}^{d}$. If $g \in I_{\Gamma}$, then $g\left(l_{i}^{d}\right)=0$ for all $i$ by $(*)$, so by linearity $g \in F^{\perp}$. Therefore $\Gamma$ is apolar to $F$.

For the converse, assume that $I_{\Gamma} \subset F^{\perp}$. Then we have surjective maps between the corresponding homogeneous coordinate rings

$$
T \rightarrow A_{\Gamma}=T / I_{\Gamma} \rightarrow A^{F} .
$$

Consider the dual inclusions of the degree $d$ part of these rings:

$$
\operatorname{Hom}\left(A_{d}^{F}, \mathbf{C}\right) \rightarrow \operatorname{Hom}\left(\left(A_{\Gamma}\right)_{d}, \mathbf{C}\right) \rightarrow \operatorname{Hom}\left(T_{d}, \mathbf{C}\right) .
$$

$D \mapsto D(f)$ generates the first of these spaces, while the second is spanned by the forms $D \mapsto D\left(l_{i}^{d}\right)$. Thus $f$ lies in the span of the $l_{i}^{d}$.

3.5. We return now to the case of cubic 4 -folds. In the notation of 1.3 we are given a general 5-dimensional space $P_{S} \subset \mathbf{P}^{14}$ defining the apolar Artinian Gorenstein ring $A^{F}$ of some cubic $F=Z(f) \subset \check{P}_{S}$. By [1] (cf. [8, 1.1]) the minimal degree of finite apolar subschemes of a general cubic 4 -fold is 10 . We first show that $F$ is general in this sense. Following 3.4 we study ideals of finite subschemes contained in $(F)^{\perp}$. Now, $(f)^{\perp}$ is generated by 15 quadrics. On the one hand these are precisely the orthogonal complement in $T_{2}$ of the partials of $F$ in $R_{2}$. On the other hand they are precisely the quadrics defining $G$ restricted to $P_{S}$. Consider the morphism $\varphi_{P}$ defined by these quadrics generating $(f)^{\perp}$. On the one hand it is the composition of the 2-uple embedding and the projection from the partials of $f$; on the other hand it is the restriction of the Cremona transformation $\varphi$ to $P_{S}$.

3.6. Lemma. The minimal degree of a finite apolar subscheme of $F$ is 10 .

Proof. A subscheme of length 9 lies on at least 12 quadrics. Therefore an apolar subscheme of length 9 would be mapped by $\varphi$ to a scheme of length 9 in a plane. If the inverse Cremona transformation restricted to this plane is birational, then the image of this plane in $\mathbf{P}^{14}$ would have degree at most 4 , so its intersection with $P_{S}$ could not be of length 9 . Therefore the plane is contained in $K^{*}$. But then the subscheme of length 9 must be contained in $G^{*}$. If the plane intersects $G^{*}$ in a conic section, then the subscheme of length 9 is contained in this conic section, i.e. in a line in $P$. This is absurd, since any apolar subscheme must span $P$. Thus the plane must be contained in $G^{*}$. There are two kinds of planes in $G^{*}$. We know 
from 2.4 that the preimage is of codimension 4 and 2, respectively, in their linear span. Again, since apolar subschemes span $P$, this is impossible.

A length 10 subscheme $\Gamma$ lies on at least 11 quadrics. Therefore if $\Gamma$ is apolar to $F$, the image $\varphi_{P}(\Gamma)$ spans at most a $\mathbf{P}^{3}$. Hence it is natural to identify a powersum of length 10 presenting $f$ with some 10 -secant $\mathbf{P}^{3}$ to $\varphi\left(P_{S}\right)$. Let $V S P_{G}(F, 10)$ be the closure in the Grassmannian $\mathbf{G}\left(4, \wedge^{2} V^{*}\right)$ of 10 -secant 3 -spaces to $\varphi\left(P_{S}\right)$. We call this a Grassmannian compactification of the set of powersums of $f$. We proceed in a few lemmas to study $V S P_{G}(F, 10)$, and in fact to show that every $\mathbf{P}^{3}$ of this family intersects $\varphi\left(P_{S}\right)$ in a finite subscheme of length 10. Therefore the Grassmannian compactification coincides with the Hilbert scheme compactification. We now make all this more precise.

Let $V S P_{G}(F, 10)$ be the closure in the Grassmannian $\mathbf{G}\left(4, \wedge^{2} V^{*}\right)$ of 3 -spaces that intersect $\varphi\left(P_{S}\right)$ in a finite subscheme of length 10 apolar to $F$.

3.7. Theorem. $V S P_{G}(F, 10)$ is isomorphic to the family of secant lines to $G^{*} \cap L_{S}$, i.e. to $\mathrm{Hilb}_{2}(S)$, where $S$ is the $K 3$ surface $S=G^{*} \cap L_{S}$.

Proof. We first define an injective map $\rho: \operatorname{Hilb}_{2}(S) \rightarrow V S P_{G}(F, 10)$. For this let $l^{*}$ be a secant or tangent line to $S$. It is defined by its intersection of length 2 with $S$. Since $S$ is general, it contains no lines, and is the intersection of quadrics, so the corresponding point in $\operatorname{Hilb}_{2}(S)$ is unique. Now, let $Q\left(l^{*}\right) \subset G^{*}$ be the quadric surface associated to $l^{*}$. According to 2.9 the preimage $\varphi^{-1}\left(Q\left(l^{*}\right)\right)$ is 7 dimensional of degree 10 and contained in $\left(l^{*}\right)^{\perp}$. But $l^{*} \subset P_{S}^{\perp}=L_{S}$ means that $P_{S} \subset\left(l^{*}\right)^{\perp}$, so $\Gamma_{l^{*}}=P_{S} \cap \varphi^{-1}\left(Q\left(l^{*}\right)\right)$ is an apolar subscheme to $F$ of length 10 as long as the intersection is proper. Clearly this is so for the general $P_{S}$ and secant line $l^{*}$. Furthermore, the image $\varphi\left(\Gamma_{l^{*}}\right)$ is always the intersection of the 3 -space $P\left(l^{*}\right)=\left\langle Q\left(l^{*}\right)\right\rangle$ with $\varphi\left(P_{S}\right)$, so the map $\rho$ is well-defined. Injectivity follows from the $1: 1$ correspondance $l^{*} \leftrightarrow P\left(l^{*}\right)$.

To show that there is a well-defined converse map we need a few lemmas. Let $\Gamma \subset P_{S}$ be an apolar subscheme to $F$, such that $\varphi(\Gamma)$ spans a $\mathbf{P}^{3}$, which we denote by $P_{\Gamma}$, and assume that $P_{\Gamma} \in V S P_{G}(F, 10)$. Thus $P_{\Gamma}$ lies on the closure of the variety of 10 -secant 3 -spaces to $\varphi\left(P_{S}\right)$. In this notation:

3.8. Lemma. Let $P_{\Gamma} \in V S P_{G}(F, 10)$. Then $P_{\Gamma}=P\left(l^{*}\right)$ for some secant or tangent line $l^{*}$ to $K^{*}$.

Proof. This proof depends on two lemmas. The first one is interesting on its own:

3.9. Lemma. If $P_{\Gamma} \in V S P_{G}(F, 10)$ as above, then $\Gamma \subset K \cap P_{S}$, and $P_{\Gamma} \subset K^{*}$.

Proof. Let $P_{\Gamma} \in V S P_{G}(F, 10)$. We may assume first that $\Gamma$ is smooth of length 10 and spans $P_{S}$. Then $\Gamma \cap G \subset P_{S} \cap G=\emptyset$, so by the Cremona transformation any point of $\Gamma$ is mapped to $G^{*}$ or to the complement of $K^{*}$. The image $\varphi(\Gamma)$ spans a 3-space $P_{\Gamma}$. The inverse Cremona transformation restricted to $P_{\Gamma}$ is defined by the quadrics through $G^{*} \cap P_{\Gamma}$. Assume that $K^{*} \cap P_{\Gamma} \neq P_{\Gamma}$. Then the restriction of the inverse Cremona transformation to $P_{\Gamma}$ is birational onto its image. Since the image intersects $P_{S}$ in $\Gamma$, which in turn spans $P_{S}$, the image must span at least an 8 -space. But in that case the degree of the image is 7 or 8 , so it cannot contain $\Gamma$. Therefore $P_{\Gamma} \subset K^{*}$, and $\Gamma \subset K$. Now, $V S P_{G}(F, 10)$ is a compactification of a set of 10 -secant $\mathbf{P}^{3}$ 's to $\varphi\left(P_{S}\right)$. Therefore any $P_{\Gamma}$ in the closure must also be contained in $K^{*}$. Furthermore the intersection $\Gamma=P_{\Gamma} \cap \varphi\left(P_{S}\right)$ must be of length at least 10. 
For 3.8 we note that $\Gamma$ is mapped to $G^{*}$ by the Cremona transformation. In the above notation we also have

3.10. Lemma. If $P_{\Gamma} \in V S P_{G}(F, 10)$, then $P_{\Gamma} \cap G^{*}$ is a quadric surface.

Proof. Consider the inverse Cremona transformation. Since $P_{\Gamma} \subset K^{*}$, the image of $P_{\Gamma} \backslash G^{*}$ is contained in $G$. By 2.2 any fiber over $G$ of the inverse Cremona transformation is a $\mathbf{P}^{5}$ which intersects $G^{*}$ in a quadric 4 -fold. Therefore the 3 space $P_{\Gamma}$ meets each fiber of this Cremona transformation in a linear space, while each of these linear spaces again intersects $G^{*}$ in at least a quadric. In the proof of 3.9 we saw that the restriction of the inverse Cremona transformation to $P_{\Gamma}$ is not birational. Therefore each fiber is a line, a plane, or all of $P_{\Gamma}$. Assume first that $P_{\Gamma}$ is contained in $G^{*}$. Then the inverse image by the Cremona transformation is a quadric hypersurface in a $\mathbf{P}^{9}$ which intersects $G$ in a Grassmannian $G(2,5)$. But $P_{S}$ does not intersect $G$, so $P_{S}$ intersects the $\mathbf{P}^{9}$ in at most a plane. In particular, $\Gamma$ is contained in a plane. But this would mean that $\Gamma$ is an apolar subscheme of $F$ contained in a plane, clearly absurd.

Therefore $P_{\Gamma}$ is not all contained in $G^{*}$. Thus $P_{\Gamma} \cap G^{*}$ is a subvariety defined by quadrics, and each fiber of the inverse Cremona transformation restricted to $P_{\Gamma}$ is a line or a plane. When the general fiber is a plane, this plane must intersect $G^{*}$ in at least a conic section, so the intersection $G^{*} \cap P_{\Gamma}$ must therefore be a quadric surface or the union of a plane and a line. On the other hand, it is not hard to check that the latter is never the intersection of a 3 -space with $G^{*}$, so if the fibers are planes then the intersection is a quadric surface.

When the fibers are lines, then $G^{*} \cap P_{\Gamma}$ is a quadric surface, the union of a plane and a line or a curve defined by quadrics with one secant line through each general point of $P_{\Gamma}$, i.e. a twisted cubic curve or the union of two lines. The union of a plane and a line never occurs as the intersection of a 3 -space with $G^{*}$, while the other cases do occur as intersections with the Grassmannian. Note that there could be no extra points of intersection in addition to these curves, since these would lie on proper trisecants to the Grassmannian, which is absurd since the Grassmannian is cut out by quadrics.

Now, the preimage under $\varphi$ of a line is a 6 -fold cubic scroll by [2.3, so the preimage of a conic section must be a 6 -fold scroll of degree 6 and the preimage of a twisted cubic curve must be a 6 -fold scroll of degree 9. But $\Gamma$ is the intersection of the 5 -space $P_{S}$ with the preimage under the Cremona transformation of $G^{*} \cap P_{\Gamma}$. If $\Gamma$ is 0-dimensional, the length of this intersection cannot exceed the degree of the corresponding preimages. Since these all have degree less than 10 , these cases are excluded and we conclude that $G^{*} \cap P_{\Gamma}$ is a quadric surface or all of $P_{\Gamma}$. If $\Gamma$ has positive dimension, then, since $\Gamma$ lies in the closure of finite schemes, $G^{*} \cap P_{\Gamma}$ is a quadric surface or all of $P_{\Gamma}$. Since the latter case is already excluded, the lemma follows.

We denote the quadric surface $P_{\Gamma} \cap G^{*}$ by $Q_{\Gamma}$. To conclude the proof of 3.8 it suffices to show that $Q_{\Gamma}$ is irreducible; but by 2.8 the quadric $Q_{\Gamma}$ is reducible only if $l^{*}$ is contained in $G^{*}$. This is excluded by our generality assumption of $S$.

For [3.7] it remains to note that $\Gamma$ is apolar to $F$ only if $P_{S} \subset\left(l^{*}\right)^{\perp}$, where $P\left(l^{*}\right)=P_{\Gamma}$.

3.11. Proposition. For a general $K 3$ surface $S$, let $F(S)$ be the associated apolar cubic 4-fold. Then the 4-folds $\operatorname{VSP}(F(S), 10)$ and $V S P_{G}(F(S), 10)$ are isomorphic. 
Proof. To prove this we have to show that for the general $K 3$ surface $S=G^{*} \cap L_{S}$, all the apolar subschemes $\Gamma$ such that the span $P_{\Gamma}$ of $\varphi(\Gamma)$ is a 3 -space contained in $\operatorname{VSP}_{G}(F, 10)$, are finite. For this we first prove the following:

3.12. Lemma. Let $P_{\Gamma} \in V S P_{G}(F, 10)$, and assume that $C$ is an integral curve contained in $\Gamma$. Then $C$ is a line.

Proof. First, note that the restriction of $\varphi$ to $P_{S}$ is an embedding defined by a linear system in $|2 h|$, where $h$ embeds $C$ in $P_{S}$. Now, $\varphi(C)$ is contained in a quadric surface by 3.10. If $\varphi(C)$ lies in a plane, then clearly $C$ must be a line. If $\varphi(C)$ lies on a quadric cone (or a smooth quadric surface) $Q_{C}$, then it meets every ruling (or every line in one ruling respectively) in at least $d$ points, where $d$ is the degree of $C$ in $P_{S}$.

Consider the preimage of a ruling of a quadric on $G$ under the Cremona transformation. In 2.3 we showed that the preimage of a line $l$ is a cubic 6 -fold scroll in a $\mathbf{P}_{l}^{8}$. When the quadric $Q_{C}$ is smooth, the intersection $\bigcap_{l} \mathbf{P}_{l}^{8}$ over all lines $l$ in the ruling $r$ is a 4 -space which we denote by $\mathbf{P}_{r}^{4}$. Furthermore, $\mathbf{P}_{r}^{4} \cap G$ is a rank 4 quadric 3-fold. When the quadric $Q_{C}$ is a cone, the intersection $\bigcap_{l} \mathbf{P}_{l}^{8}$ is the 5-space $\mathbf{P}_{r}^{5}=\varphi^{-1}(v)$, where $v=\operatorname{Sing} Q_{C}$ is the vertex of $Q_{C}$, and the intersection $\mathbf{P}_{r}^{5} \cap G$ is a smooth quadric. Now, if $\varphi(C)$ intersects each line $l$ in the ruling $r$ in $a$ points, then $C \cap \mathbf{P}_{l}^{8}$ contains at least $a$ points. If $a>d$, this means that $C \subset \mathbf{P}_{l}^{8}$ for each line $l$ in the ruling. So $C$ is contained in the intersection $\mathbf{P}_{r}^{4}$ or $\mathbf{P}_{r}^{5}$. In either case $C$ must intersect $G$, a contradiction.

Therefore $\varphi(C)$ meets every ruling in at most $d$ points, i.e. it must be a complete intersection of the quadric surface with a surface of degree $d$, and $g(C)=(d-1)^{2}$. If $C$ is a plane curve, it is clearly a line. Otherwise, Castelnuovo's bound for space curves applies and $g(C)=(d-1)^{2} \leq \frac{1}{4} d^{2}-d+1=\left(\frac{d}{2}-1\right)^{2}$, which is possible only if $d=1$.

Let $\mathcal{F}(K)$ be the 22 -fold of lines on the Pfaffian cubic 13 -fold $K \subset \mathbf{P}^{14}$, and let $G(6,15)$ be the Grassmannian of 5 -dimensional projective subspaces $P_{S} \subset \mathbf{P}^{14}$. Any line $l \in \mathcal{F}(K)$ is mapped to a conic section $\varphi(l) \subset \check{\mathbf{P}}^{14}$ by the Cremona transformation. On the other hand, by [2.7] there is a unique common Lagrangian 4-space $V^{*}(l) \subset V^{*}$ for all $g \in l$. When $l \subset P_{S} \cap K$, then $\mathbf{P}\left(\wedge^{2} V^{*}(l)\right)$ intersects $L_{S}=P_{S}^{\perp}$ in a line $l^{*}$, which is neccessarily a secant line to $G^{*}$, therefore also contained in $K^{*}$. In fact, let $P\left(l^{*}\right) \subset \mathbf{P}\left(\wedge^{2} V^{*}(l)\right)$ be the 3 -space associated to the secant line $l^{*}$ to $G^{*}$ as in 2.8 (i.e. the polar $\mathbf{P}^{3}$ to $l^{*}$ with respect to the quadric $\left.\mathbf{G}\left(2, V^{*}(l)\right)\right)$. It follows from 2.9 that the preimage of $P\left(l^{*}\right)$ under the Cremona transformation is contained in the complement $l^{* \perp} \subset \mathbf{P}^{14}$. But $l^{*} \subset L_{S}=P_{S}^{\perp}$, so $P_{S} \subset l^{* \perp}$. If the intersection between $P_{S}$ and the fiber is proper inside $l^{* \perp}$, then it is a finite subscheme of length 10 . In other words, $P\left(l^{*}\right)$ is a 10 -secant $\mathbf{P}^{3}$ to $\varphi\left(P_{S}\right)$ for general $l \subset P_{S}$.

It remains to show that for general $P_{S}$ the intersection is always proper. Now clearly $\varphi(l) \subset \mathbf{P}\left(\wedge^{2} V^{*}(l)\right)$, since $g \in l$ implies that $V^{*}(l)$ is a Langrangian 4-space with respect to $g$, i.e. $\operatorname{ker} g \subset V^{*}(l)$. Therefore $l \subset \Gamma$ only if $\varphi(l) \subset P\left(l^{*}\right) \subset$ $\mathbf{P}\left(\wedge^{2} V^{*}(l)\right)$.

Consider the incidence

$$
I=\left\{\left(l, P_{S}\right) \mid l \subset P_{S}, \varphi(l) \subset P\left(l^{*}\right)\right\},
$$


and let $p: I \rightarrow \mathcal{F}(K)$ and $q: I \rightarrow \mathbf{G}\left(6, \wedge^{2} V\right)$ be the natural projections. The incidence naturally parametrizes the bad locus, i.e. the set of spaces $P_{S}$ such that there exist some $P_{\Gamma} \in V S P_{G}(F, 10)$ where $\Gamma$ contains a line. Therefore it is enough to show that the second projection is not surjective. In fact we will show that the codimension of $q(I)$ in $\mathbf{G}\left(6, \wedge^{2} V\right)$ is at least 2 .

Let $l \subset K$ be a general line; in particular, $l \subset K \backslash G$, Then

$$
p^{-1}(l)=\left\{P_{S}=\mathbf{P}^{5} \subset \mathbf{P}^{14} \mid l \subset P_{S}, \varphi(l) \subset P\left(l^{*}\right)\right\} .
$$

But $\varphi(l)$ and $P\left(l^{*}\right)$ are both subvarieties of $\mathbf{P}\left(\wedge^{2} V^{*}(l)\right)$. The cycle of 4 -spaces that contain the conic section $\varphi(l)$ is a plane inside $G\left(4, V^{*}(l)\right)$, so it is of codimension 6 in the Grassmannian and the fiber $p^{-1}(l)$ is of codimension 6 among all $P_{S}$ that contain $l$, i.e. the fiber has dimension 30 . On the other hand, the family of lines in the Pfaffian cubic $K$ is of dimension 22, while $\mathbf{G}\left(6, \wedge{ }^{2} V\right)$ has dimension 54 . Therefore the image of $q$ has codimension at least 2 .

3.13. Corollary. Let $P_{S}$ be general. Then $\operatorname{VSP}(F(S), 10)$ is isomorphic to the family of secant lines to $G^{*} \cap L_{S}$, i.e. to $\operatorname{Hilb}_{2}(S)$, where $S$ is the K3 surface $S=G^{*} \cap L_{S}$. Furthermore, $\operatorname{VSP}(F(S), 10)$ is isomorphic to the Fano variety of lines $\mathcal{F}\left(F^{\prime}\right)$ of the Pfaffian cubic fourfold $F^{\prime}=F^{\prime}(S)=P_{S} \cap K$, where $P_{S}=L_{S}$.

Proof. The first statement follows directly from 3.7 and 3.11. The argument following the proof of 3.12 shows that $\operatorname{Hilb}_{2}(S)$ is isomorphic to the Fano variety of lines in $F^{\prime}(S)$, as was noticed by Beauville and Donagi (cf. [2]).

3.14. The corollary suggests an incidence correspondence

$$
I=\{(g, \Gamma) \mid g \in \Gamma\} \subset F^{\prime}(S) \times \operatorname{VSP}(F(S), 10) \cong F^{\prime} \times \mathcal{F}\left(F^{\prime}\right) .
$$

The second projection is clearly $10: 1$.

3.15. Proposition. The projection of the incidence correspondence

$$
I \subset F^{\prime}(S) \times V S P(F(S), 10)
$$

onto the first factor is generically $6: 1$.

Proof. Consider a general point $g \in F^{\prime}(S)=P_{S} \cap K$. Since $g \in K \backslash G$, the Cremona transformation $\varphi$ is defined in $g$ and the fiber containing $g$ is a 5 -space $P_{g}=P\left(\wedge^{2}|g|\right)$. The space $P_{g}$ intersects $G$ in a quadric $Q_{g}$ corresponding to all lines in the 3 -space $P(|g|)$, and $|g|=\operatorname{ker} \varphi(g)$. In the dual space, $P_{g}^{\perp} \subset \check{\mathbf{P}}^{14}$ is the tangent 8 -space $T_{\varphi(g)}$ to $G^{*}$ at $\varphi(g)$. Now, the point $p$ is in the image of the projection from the incidence correspondence, i.e. is contained in some $\Gamma \in \operatorname{VSP}(F(S), 10)$, if $P_{g}$ is contained in two special tangent hyperplanes, corresponding to two points on $G^{*}$. These special tangent hyperplanes which contain $P_{g}$ are parametrized by all 3-spaces that intersect $P(|g|)$ in a plane. On $G^{*}$ this is, by 2.5. precisely what is defined by the minors of a matrix $N_{g}$ equivalent to $N_{01}$ inside the tangent space $T_{\varphi(g)}=P_{g}^{\perp} \subset \check{\mathbf{P}}^{14}$. The hyperplanes which contain both $P_{S}$ and $P_{g}$ form $P_{S}^{\perp} \cap P_{g}^{\perp}$ in $\check{\mathbf{P}}^{14}$. This is a 3 -space, since the two 5 -spaces $P_{S}$ and $P_{g}$ intersect. Inside $T_{\varphi(g)}$ the minors of $N_{g}$ define a rational quartic 5-fold scroll, so the intersection with $L_{S}=P_{S}^{\perp}$ is 4 points. The secants between the 4 points are secant lines to $L_{S} \cap G^{*}$, corresponding to sets $\Gamma$ that contain $g$. 
3.16. Finally, we turn to the general cubic 4-fold. We want to show that $\operatorname{VSP}(F(S), 10)$ deforms smoothly with $F$, or more precisely that $\operatorname{VSP}(F, 10)$ for a general cubic 4-fold $F$ is a deformation of $\operatorname{VSP}(F(S), 10)$. But $\operatorname{VSP}(F(S), 10)$ is a symplectic manifold, and its space of deformations is smooth of dimension 20 (cf. [2]). In fact every general member of this deformation is the Fano variety of lines in some cubic 4 -fold. Since the variety of cubic fourfolds has dimension 20, it would follow that

3.17. Theorem. $\operatorname{VSP}(F, 10)$ for a general cubic fourfold $F$ is isomorphic to the Fano variety of lines in some other cubic fourfold.

Proof. Consider the correspondence

$$
V S P=\{(\Gamma, F) \mid \Gamma \in V S P(F, 10)\} \subset \operatorname{Hilb}_{o}^{10} \mathbf{P}^{5} \times \mathbf{P}\left(\operatorname{Sym}^{3} V\right)
$$

where $\mathbf{P}^{5}=\mathbf{P}(V)$, and $\operatorname{Hilb}_{o}^{10} \mathbf{P}^{5}$ is the component of the Hilbert scheme that contains the smooth subschemes $\Gamma$ that span $\mathbf{P}^{5}$. The fiber of the second projection is $\operatorname{VSP}(F, 10)$ for a cubic $F \in \mathbf{P}\left(\operatorname{Sym}^{3} V\right)$. The fiber over a point $\Gamma \in \operatorname{Hilb}_{o}^{10} \mathbf{P}^{5}$ by the first projection is a linear space, the span $\mathbf{P}(\Gamma)$ of $\rho_{3}(\Gamma)$ in $\mathbf{P}\left(\operatorname{Sym}^{3} V\right)$ under the 3 -uple embedding $\rho_{3}$. For the general $\Gamma$ the span $\mathbf{P}(\Gamma)$ is of course a $\mathbf{P}^{9}$, while Hilb $_{o}^{10} \mathbf{P}^{5}$ has dimension 50, so $V S P$ is reduced of dimension 59. The group $G L(V)$ acts on both factors, and on the incidence $V S P$. Since the general cubic and the general subscheme $\Gamma$ have no nontrivial automorphisms, the quotient by $G L(V)$ is smooth at the general point, and the general fiber of the second projection is the same after taking quotients. Thus for our purposes it suffices to check the second projection in the above incidence.

By 3.13 the general fibers of the second projection over the hypersurface in $\mathbf{P}\left(\mathrm{Sym}^{3} V\right)$ of apolar cubic 4-folds $F(S)$ are smooth 4-dimensional symplectic varieties. Consider a general point $(\Gamma, F(S))$ in one of these fibers.

3.18. Lemma. The projection $V S P \rightarrow \mathbf{P}\left(\operatorname{Sym}^{3} V\right)$ has maximal rank at $(\Gamma, F(S))$.

Proof. We may assume that $\Gamma$ is smooth, i.e. that $\Gamma=\left\{l_{1}, \ldots, l_{10}\right\} \subset \mathbf{P}(\mathrm{V})$ and that $f=l_{1}^{3}+\ldots+l_{10}^{3}$, where $F(S)=Z(f)$. The rank of the map onto $\mathbf{P}\left(\operatorname{Sym}^{3} V\right)$ is then the dimension of the span of $\left\{l_{i}^{2} y_{j} \mid 1 \leq i \leq 10,0 \leq j \leq 5\right\}$, where $\left\langle y_{0}, \ldots, y_{5}\right\rangle=V$. In fact, from the expansion of $\left(l_{i}+y_{j}\right)^{3}$, we see that $l_{i}^{2} y_{j}$ defines a tangent direction at the point $l_{i}^{3}$, so the above span is the span of the tangent spaces to the 3 -uple embedding $\rho_{3}(\mathbf{P}(V))$ at the points $l_{i}^{3}$. By Terracini's lemma (cf. [10]) the tangent space to the 10th secant variety of $\rho_{3}(\mathbf{P}(V))$ at $F(S)$ is precisely the span of the tangent spaces at the 10 points $l_{i}^{3}$. If the span is not all of $\mathbf{P}\left(\operatorname{Sym}^{3} V\right)$, there is a hyperplane section of $\rho_{3}(\mathbf{P}(V))$ singular at the 10 points, i.e. a cubic hypersurface in $\mathbf{P}(V)$ singular in the points $l_{1}, \ldots, l_{10}$. But it follows from 2.9 that $\left\{l_{1}, \ldots, l_{10}\right\}$ is a general intersection of two rational quartic scrolls in a quadric hypersurface. By genericity it is enough to check in one example that such a subscheme is not the singular locus of any cubic hypersurface. In fact the two matrices

$$
\left(\begin{array}{llll}
x_{0} & x_{2} & x_{4} & x_{0}+x_{5} \\
x_{1} & x_{3} & x_{5} & x_{3}+x_{4}
\end{array}\right) \text { and }\left(\begin{array}{llll}
x_{0} & x_{1} & x_{1}+x_{2} & x_{1}+x_{5} \\
x_{2} & x_{3} & x_{4}-x_{5} & x_{0}+x_{3}
\end{array}\right)
$$

have a common $2 \times 2$ minor. It is easy to check with MACAULAY [6] that the two matrices drop rank along 10 points, and that there are no cubic hypersurface singular along these points. Thus the lemma follows. 
The point $(\Gamma, F(S))$ is therefore a regular point for the projection $V S P \rightarrow$ $\mathbf{P}\left(\operatorname{Sym}^{3} V\right)$, i.e. the fibration is smooth at this point. Since the fiber itself is smooth, the general fiber is smooth, and is a deformation of $\operatorname{VSP}(F(S), 10)$.

Theorem 3.17 describes a map $\mu$ from an open set of the moduli of cubic 4-folds into itself. By 1.6 this map is not the identity. Therefore we pose

3.19. Problem. Is $\mu$ dominant, and if so, what is its degree?

\section{REFERENCES}

[1] Alexander, J., Hirschowitz, A.: Polynomial interpolation in several variables, J. of Alg. Geom. 4 (1995), 201-222. MR 96f:14065

[2] Beauville, A., Donagi, R.: La variete des droites d'une hypersurface cubique de dimension 4. Compt. Rendu. Acad. Sc. Paris. 301 (1986) 703-706. MR 87c:14047

[3] Ein, L., Shepherd-Barron, N.: Some special Cremona transformations, Amer. J. Math. 111 (1989) 783-800. MR 90j:14015

[4] Eisenbud, D.: Commutative Algebra with a View Toward Algebraic Geometry. GTM 150 Springer-Verlag, New York, 1995. MR 97a:13001

[5] Macaulay, F.S.: Algebraic theory of modular systems. Cambridge University Press, London, (1916). MR 95i:13001 (rev. reprint)

[6] Bayer, D., Stillman, M.: MACAULAY: A system for computation in algebraic geometry and commutative algebra, Source and object code available for Unix and Macintosh computers. Contact the authors, or download from zariski.harvard.edu via anonymous ftp.

[7] Mukai, S.: Curves, K3 surfaces and Fano 3-folds of genus $\leq 10$, in "Algebraic Geometry and Commuatative Algebra in Honor of Masayoshi Nagata", pp. 357-377, (1988), Kinokuniya, Tokyo. MR 90b:14039

[8] Ranestad, K., Schreyer, F-O: Varieties of sums of powers, to appear in J. Reine Angew. Math. (2000).

[9] Salmon, G.: Modern Higher Algebra, 4. Edition. Hodges, Figgis, and Co., Dublin (1885)

[10] Zak, F. L.: Varieties of small codimension arising from group actions. Addendum to Lazarsfeld and Van de Ven: Topics in the Geometry of Projective Space, DMV Seminar 4 (1984). MR 87e:14045

Institute of Mathematics, Bulgarian Academy of Sciences, Acad. G. Bonchev Str., 8, 1113 Sofia, Bulgaria

E-mail address: ailiev@math.bas.bg

Matematisk Institutt, UiO, P.B. 1053 Blindern, N-0316 Oslo, Norway

E-mail address: ranestad@math.uio.no 